\title{
Erratum to: Nationwide analysis on surgical procedures for patients with endometrial cancer in Germany: Results of the AGO pattern of care studies from the years 2013, 2009, and 2006
}

\author{
Marco Johannes Battista $\cdot$ Marcus Schmidt $\cdot$ Nicole Rieks $\cdot$ Joscha Steetskamp \\ Isabel Sicking • Antje Lebrecht $\cdot$ Heinz Koelbl $\cdot$ Peter Mallmann • \\ Gerald Hoffmann · Eric Steiner
}

Published online: 19 August 2014

(C) Springer-Verlag Berlin Heidelberg 2014

\section{Erratum to: J Cancer Res Clin Oncol}

DOI 10.1007/s00432-014-1755-7

Unfortunately, the initial sentence in the "Conclusions" section of the Abstract was incorrectly published. The correct sentence should read:

The paraaortic LAN, the LSA as well as the second operation on patients who had postoperatively been upstaged were not conducted in accordance with the guideline.

The online version of the original article can be found under doi:10.1007/s00432-014-1755-7.

M. J. Battista $(\bowtie) \cdot$ M. Schmidt $\cdot$ N. Rieks $\cdot$ J. Steetskamp .

I. Sicking $\cdot$ A. Lebrecht $\cdot$ G. Hoffmann

Department of Gynecology and Obstetrics,

University Hospital Mainz, Mainz, Germany

e-mail: battist@uni-mainz.de; Battist@mail.uni-mainz.de

H. Koelbl

Department of Gynecology and Gynecological Oncology,

General Hospital (AKH), Medical University of Vienna,

Vienna, Austria

P. Mallmann

Department of Gynecology and Obstetrics,

University Hospital Köln, Köln, Germany

P. Mallmann · E. Steiner

Disease Committee Uterine Neoplasm of the Arbeitsgemeinschaft

Gynäkologische Onkologie (AGO), Taufingen, Germany

E. Steiner

Department of Gynecology and Obstetrics,

GP Ruesselsheim, Ruesselsheim, Germany 